\title{
Minimum cross-entropy reconstruction for synthetic aperture radar imagery
}

\author{
M. GRIPPA ${ }^{1}$ and I. H. WOODHOUSE
}

School of GeoSciences, The University of Edinburgh, Edinburgh EH8 9XP, U.K.

\begin{abstract}
Speckle limits the quantitative quality of SAR imagery and makes performing post-processing tasks difficult. It is therefore important to develop algorithms that can remove or minimise the influence of speckle while preserving the original data.

In this paper we analyse the applicability of minimum cross-entropy methods for SAR image reconstruction. Our novel approach focuses on the form of the goodness-of-fit function for which we also employ an entropy function in addition to the penalty function. Such an approach seems more suited to the multiplicative nature of speckle. An adaptive minimum cross-entropy (MCE) algorithm is developed on this basis and its performance is investigated on both simulated and real SAR data. The results indicate a general level of performance comparable to commonly available reconstruction techniques such as those available in ENVI and CAESAR as well as a more traditional form of the entropy formulation. The method developed is found to be particularly well suited for images of natural landscapes containing a distribution of features and textures.
\end{abstract}

\section{Introduction}

SAR images are affected by speckle, a noise-like pattern originating from interference of scattered waves, usually modelled as a multiplicative noise that has been imposed upon an underlying radar cross-section (Oliver and Quegan 1998). For most application the potential of SAR images can be greatly enhanced by reducing the influence of speckle. A commonly used technique to achieve this is multi-look processing that reduces speckle variance by a factor $L$ at the expenses of spatial resolution that is worsened by the same factor. Several algorithms have been developed in the past to achieve speckle reduction while retaining edge sharpness and preserving the image features. This has been done, for example, using different kinds of filters (Oliver and Quegan 1998, Lee and Jurkevich 1994) or applying Bayesian inference methods to reconstruct the radar cross-section underlying the noisy data (Datcu et al. 1998). Due to the noise-like ambiguity introduced by speckle the inverse problem of reconstructing the original cross section does not have a unique solution and some additional criteria is

\footnotetext{
${ }^{1}$ now at CESBIO, 18, Av. Edouard Belin, 13401 Toulouse Cedex 09, France, tel:+33(0)561558584, fax:+33(0)561558500, email:manuela.grippa@cesbio.cnes.fr
} 
necessary to select the best solution among all those that are possible within the measurement uncertainty. This is often done by including some a priori information independent on the measurements.

Maximum Entropy (ME) and Minimum Cross Entropy (MCE) methods are based on the employment of an entropy function to describe the a priori information (Jaynes 1982). These techniques have been successfully applied to a wide range of inverse problems, including image reconstructions (e.g. Burc et al. 1983, Gull 1989) where they have been demonstrated to be capable of preserving details in the reconstructed image even in presence of considerable noise. However, they have not been widely used in remote sensing and they have not received much attention for SAR applications (with some exceptions i.e. Datcu et al. 1998, Lopes and Sery 1997).

The aim of this paper is to perform a preliminary investigation on the performance of a MCE method for SAR image reconstruction and despeckling. We deliberately keep the algorithm simple (for example no edge directed windows are employed) in order to investigate the potential of this technique at a fundamental level rather than developing an optimal filter at this stage. In section 2 we briefly review concepts of minimum cross-entropy methods, describing both the traditional formulation and our novel approach based on the employment of an entropy function for the goodness-of-fit function. Then, we introduce a filter-based approach for the MCE algorithm that uses information derived from local image statistics to set the relative weight between measurements and prior information (hence the name "adaptive MCE"). Section 3 describes the criteria adopted to evaluate the performance of the MCE methods and the commercially available speckle filters we employ to compare our results. The results obtained on simulated SAR images are reported in section 4: first, the performance of the MCE applied as a global minimisation procedure is discussed. Then, the results achieved by applying the adaptive MCE are reported and evaluated through a comparison with those achieved by commercially available filters. To conclude, some examples of real airborne and spaceborne SAR data reconstructions are shown (section 5). Perspectives, problems and further developments of this work are addressed in the concluding discussion.

\section{Minimum cross-entropy methods}

Due primarily to the presence of speckle, the original observations within an image, $\mathbf{z}$, are not unique so that practical inverse methods have to provide some criteria to choose the best solution through the inclusion of some a priori information. One approach is to use a penalty function, expressed as $\Delta_{2}\left(\mathbf{x}, \mathbf{x}^{0}\right)$, that 
incorporates the constraint on the solution $\mathrm{x}$ relative to some prior estimate $\mathrm{x}^{0}$. The solution is then chosen by minimising a function $\Delta_{1}(\mathbf{x}, \mathbf{z})$ expressing the fit of the reconstructed image to the measurements subject to the penalty function $\Delta_{2}\left(\mathbf{x}, \mathbf{x}^{0}\right)$, i.e. by minimising the following expression:

$$
Q(\mathbf{x})=\Delta_{1}(\mathbf{x}, \mathbf{z})+\lambda \Delta_{2}\left(\mathbf{x}, \mathbf{x}^{0}\right) .
$$

where $\lambda$ is the parameter that controls the relative weight of the two terms. ME and MCE methods are based on the employment of an entropy function for $\Delta_{2}\left(\mathbf{x}, \mathbf{x}^{0}\right)$. The specific advantages of using this kind of approach are that:

- the solution is always positive;

- entropy should allow fine details and edges in the solution;

- entropy methods work well with high dynamic range.

There is also an argument that MCE methods result in the least committal solution given the available data (i.e. they keep to a minimum the prior assumption). Therefore, they can be assigned an unique position, among image reconstruction methods, by their relation with the Jaynes Principle of Maximum Entropy (Jaynes 1982).

\subsection{Global MCE minimisation}

The solution is obtained by minimising $Q(x)$ in equation (1) globally, i.e. on the entire image.

In this paper we adopt the following cross entropy term for the penalty function:

$$
\Delta_{2}\left(\mathbf{x}, \mathbf{x}^{0}\right)=\sum_{i=1}^{N} \frac{x_{i}}{\sum_{j=1}^{N} x_{j}}\left(\log \frac{x_{i}}{\sum_{j=1}^{N} x_{j}}-\log \frac{x_{i}^{0}}{\sum_{j=1}^{N} x_{j}^{0}}\right) .
$$

where $\frac{x_{i}}{\sum_{j=1}^{N} x_{j}}$ is the normalised pixel value at position $i$.

In many published works on entropy methods, the goodness-of-fit function $\Delta_{1}(\mathbf{x}, \mathbf{z})$ is determined by assuming a $\chi^{2}$ statistical distribution (referred as MCE$\chi^{2}$ method from now onwards):

$$
\Delta_{1}(\mathbf{x}, \mathbf{z})=\chi^{2}=\sum_{i=1}^{N} \frac{\left(x_{i}-z_{i}\right)^{2}}{e_{i}^{2}}
$$

where $e_{i}$ is the expected uncertainty on measurement $z_{i}$. Equation 3 is derived under the assumption that the set of measurements have independent Gaussian 
errors in which case the expected value of the $\chi^{2}$ distribution is equal to the number of data values $N$. Although this method is widely used, it has been criticised by several authors (e.g. Gull 1989): the condition $\chi^{2}=N$ is only valid for an average over a large number of repeated measurements (i.e. in our case only for large areas of uniform radar cross-section) and in general it leads to under-fitting. Moreover, radar imagery with few number of looks are nearly all noise so that $\chi^{2}=N$ for any value of $\lambda$. Finally, this condition relies on an accurate estimate of the uncertainty $e_{i}$ in the measurements that is not often well known.

Therefore we consider a new form for $\Delta_{1}(\mathbf{x}, \mathbf{z})$ that is also based on an entropy function (MCE-MCE method):

$$
\Delta_{1}(\mathbf{x}, \mathbf{z})=\sum_{i=1}^{N} \frac{x_{i}}{\sum_{j=1}^{N} x_{j}}\left(\log \frac{x_{i}}{\sum_{j=1}^{N} x_{j}}-\log \frac{z_{i}}{\sum_{j=1}^{N} z_{j}}\right) .
$$

This gives a consistent overall approach to problems where the image statistics are not well known or difficult to characterise and it is expected to give improved results for high noise configurations. This function is also related to the ratio of the arithmetic to the geometric mean, often used in segmentation algorithms (Woodhouse 2001).

Since we are not considering a kernel for the image model, smoothing, and therefore speckle reduction, is only provided by the a priori. The incoherent system point spread function can be used as kernel as in (Datcu 1998) but it is often difficult to characterise. In the following analysis we consider a local a priori by passing a boxcar filter on the noisy image. The size of the moving window on which the mean filter is calculated can be chosen as the value that gives the optimum trade-off between noise reduction and spatial averaging. This optimum value can also be derived using a minimum cross-entropy approach (Grippa and Woodhouse 2002a ).

\subsection{Adaptive Minimum Cross-Entropy}

To decrease the computational time required by the global MCE minimisation and to reduce the complexity of the problem we adopt a filter-based approach in which cross-entropy is locally minimised. We found that the results obtained performing the minimisation of $Q(x)$ on smaller square windows (up to $4 \times 4$ pixels wide) do not significantly change the reconstruction results ${ }^{2}$ while speeding up considerably the algorithm.

\footnotetext{
${ }^{2}$ We suspect this happens because we do not employ any kernel to model the image formation so we do not introduce correlation amongst pixels. Different conclusion may be reached otherwise.
} 
Also we develop an adaptive MCE method by assigning the values of $\lambda$ according to the local statistics of each of the windows in which cross-entropy is locally minimised. These values are calculated from the ratio of the arithmetic and geometric mean (A/G). A/G has been widely used in the past as an edge detector and in its logarithmic form it has been demonstrated to express the additional information brought to the data by constraining it to be constant and equal to the mean (Woodhouse 2001). Our aim is to constrain the data toward the prior more strongly in uniform regions (the prior is the mean filter, which for regions of uniform radar cross section is considered optimum) than in areas of large intensity variation (such as edges). This can be achieved by putting:

$$
\lambda_{k}=\frac{1}{<\log \mathrm{A} / \mathrm{G}>_{\mathrm{k}}},
$$

where $\langle. .\rangle_{k}$ represents the mean value over the window $k$ in which the crossentropy is locally minimised. An example of the values of $\lambda$ calculated as in equation (5) for a test image is reported in figure 1: the edge areas are well individuated by low values of $\lambda$ in which case the reconstruction is not significantly biased by the prior.

\section{Performance evaluation}

\subsection{Criteria employed in the evaluation}

For simulated images, the overall performance of the methods investigated can be evaluated by the mean square errors (MSE) between the original image (without noise) and the reconstructed image. However, the MSE cannot be calculated for real SAR images and other parameters have to be considered as quality indicators. In this paper we use the following criteria.

- Retention of the mean values (Mean): the mean value of the reconstructed images should equal to that of the original image.

- Speckle reduction (Speckle): the equivalent number of looks for the intensity ratio image should be close to $L$ (Walessa and Datcu 2000).

- Edge sharpness (Edge). From known edges the means are measured along 3 pixels wide strips adjacent to the edges. The absolute difference in the means is taken as a measure of edge sharpness (Lee and Jurkevich 1994).

\subsection{Standard despeckling methods used to compare to the MCE methods}


Here we compare our results to those obtained by applying other standard RCS reconstruction methods such as those commercially available ${ }^{3}$ in ENVI (routine gamma (Shi and Fung, 1994)) and CAESAR (routine gammann (McConnell and Oliver, 1996)). In these algorithms the RCS reconstruction is performed by solving an optimisation problem to find the best fit of a given model to the image data. Both the ENVI and the CAESAR gamma routines assume a gamma distribution for the RCS model. CAESAR employs a variant of the simulated annealing technique to perform the optimisation.

Segmentation methods are considered to be the current state-of-art for many SAR image despeckling applications. Even if the MCE method developed in this paper is a RCS reconstruction method, based on a pixel by pixel estimation of the RCS, we also compare its performance to those achieved by a segmentation algorithm, based on a spatial estimation of the RCS. Segmentation methods rely on the cartoon model that assumes images are made up of regions, separated by edges, in which some parameter is constant. The cartoon model assumption is ideal for images containing sharp boundaries such as man-made landscapes or natural features such as the edges of woodland, river and lakes and the edges of flooded or burnt areas. The segann routine in CAESAR, employed in this study, produces cartoon images looking for regions of constant underlying RCS. Pixels located at the edges are transferred from one region to another according to the likelihood that the segmentation result fits the image data. This process is governed by simulated annealing and it will eventually find the global optimum solution for the segmentation of the image into a specified number of regions.

\section{Results on simulated SAR images}

\subsection{Global MCE reconstruction}

In this section we present the result of the MCE reconstruction method described in section 2.1. We consider two test images with different information content (figure 2 (a) and (d)) to which we added simulated random speckle noise. The corresponding images with added simulated noise equivalent to 6-looks are reported in figure 2 (b) and (e). The results of the MCE-MCE reconstruction method are shown in figure 2 (c) and (f).

Table 1 reports the values for mean retention, speckle reduction, edge sharpness and MSE for the two test images in figure 2 and for their reconstructions

\footnotetext{
${ }^{3}$ More advanced despeckling programs, as for example the fi lter reported by Lee and Jurkevich (1994), may also be employed for a validation of the method suggested. However our aim here is to provide a reference for a comparison with algorithms that are widely available to everyone.
} 
achieved using our novel MCE approach (MCE-MCE) and the more traditional MCE formulation (MCE- $\chi^{2}$ ). The results achieved applying the MCE-MCE method are slightly better than those obtained with the MCE- $\chi^{2}$ approach, in terms of edge preservation (the edge reported values are closer to the original ones) and MSE values (the MSE values are lower). Results for the mean filter, that constitutes the prior information, are also reported. In both cases (test images A and B) the MCE approach provide an improvement over the prior: speckle is reduced and the edges are better preserved. The lowest values of the mean square errors are obtained for the MCE-MCE method for both images.

An analogous behaviour to that described here has been found for different noise configurations (e.g. results obtained with added noise equivalent to 3-looks are reported in Grippa and Woodhouse, 2002b).

From the analysis reported above the MCE-MCE method seems to have good potential for SAR image reconstruction. Also, this method has been found to perform well in comparison with other basic standard speckle filters (such as the basic Lee filter and the Frost filter (Grippa and Woodhouse 2002b)).

However two main problems need to be further addressed.

1. Performing a global cross-entropy minimisation is computationally too demanding and the mathematical and algorithm complexity of the problem greatly increase with the image size. The method described above is not operatively applicable to the reconstruction of medium/large images.

2. The results of the minimisation are dependent on the choice of the parameter $\lambda$, that controls the bias of the prior information. In the above analysis this has been assigned a posteriori on the basis of the performance achieved in terms of speckle reduction: this estimation is effective but it requires iteration on the values of $\lambda$ that further slows the process.

In the following section we show the improved results obtained by the application of the adaptive MCE method (section 2.2) that overcomes the above problems.

\subsection{Adaptive MCE}

The reconstruction results obtained by applying the adaptive MCE method on the test images in figure 2 are shown in figure 3 (a) and (d). The corresponding values for mean retention, speckle reduction, edge preservation and MSE are reported in table 2. A visual analysis of the images in figure 2 and 3 and a comparison between the values in tables 1 and 2 make apparent that the adaptive MCE 
method is more effective in preserving edges and small features but marginally less efficient in reducing speckle than the MCE-MCE method discussed in the previous section. The values of MSE are lower for the adaptive MCE and an overall improvement is achieved.

Figure 3 and table 2 also report the results achieved using the gamma filter in ENVI and in CAESAR, and the CAESAR segmentation algorithm. The reconstructed images obtained with the gamma filter in ENVI (figure 3 (b) and (f)) present a better edge preservation than those achieved using the MCE method (see also the values in table 2) but they are not very good for speckle reduction. This is evident especially in the vicinity of the edges where little of no reconstruction is performed. The high noise around the edge areas is reflected in high values of the MSE. The CAESAR gammann routine is more efficient in despeckling but sometimes an excessive smoothing is produced which causes loss of texture information (as in the case of test image B in figure $3(\mathrm{~g})$ to be compared with the original image in figure 2(d)). For both test images the lowest values of the MSE are obtained for the adaptive MCE reconstruction (see tables 2 and 1).

Regarding the comparison with the segmentation results, in the case of test image A (figure 3(d)), which is ideal for the cartoon model, segmentation proves to be better than the other RCS reconstruction methods, giving by far the lowest value of the MSE and better values regarding the other metrics considered in the evaluation. In the case of the natural landscape in test image B, segmentation produces very good results in respect to edge preservation and the retention of the mean value. However the resulting image is very smooth (see figure $3(\mathrm{~h}$ ) and the ENL values in table 2) and the texture properties of the forested area have been lost in the segmentation process. The overall values of the MCE are lower when using the adaptive MCE reconstruction. This is to be expected since the segmentation assumes a cartoon model, which is not so appropriate for a natural scene of mixed targets.

\subsection{Performance dependence on the number of looks}

Figure 4 reports the values of the root mean square errors between the original test images A and B and their reconstruction using the Adaptive MCE, ENVI and CAESAR for different configuration of the simulated added noise (corresponding to a number of looks ranging between 1 and 30). Among the RCS reconstruction methods, the MCE approach produces the lowest values of the RMSE for images with few number of looks, i.e. with high noise, as expected from the theoretical formulation of the problem. Increasing the number of looks, better results are obtained with ENVI and CAESAR: this is more evident for the test image A while 
for the natural landscape image (test B) lower values of the RMSE are obtained using the MCE method for $L$ up to a value of 18 .

Segmentation is superior to the RCS methods employed in this analysis in the case of test images A: the lowest values of RMSE are obtained for all the range of L. This is not surprising giving the nature of image A, which is particularly suited to a segmentation approach. For test image B, the segmentation results are better than the RCS reconstruction results for L up to 3. In this case the efficiency in despeckling provide the lowest values of the MSE. For values of L higher than 3 the MCE method perfoms better than the CAESAR segmentation and for high values of L all the RCS methods give lower values of the MSE: in this case the speckle reduction provided by segmentation does not compensate the loss of information content caused by the excessive smoothing of the natural features.

\section{Results on SAR data}

To conclude we discuss two examples of reconstruction on real SAR data: airborne and spaceborne.

Figure 5(a) shows an high-resolution SAR image (L-band, HH polarisation, 4-looks) acquired over Glen Affric, Scotland, during the SHAC-UK campaign by the E-SAR sensor on board the DLR aircraft. The adaptive MCE reconstruction is reported in figure 5(b). The lower image in the same figure shows a photograph of the same area, with a dense pine plantation (apparent in the radar image as the bright triangle at the top) and a mixture of bare and low vegetation covered surfaces. A detail of the reconstruction is shown in figure 6 in comparison with the images obtained using ENVI and CAESAR: as for the case studies reported in the previous section, ENVI appears less effective in speckle reduction and rings of noise are apparent across the plantation area. On the other hand some texture loss is apparent for the reconstructed image obtained by CAESAR using both the RCS reconstruction and the segmentation routines. The image obtained using the adaptive MCE algorithm seems to preserve the texture of the pine plantation best while reducing the speckle noise.

A quantitative evaluation of the results (calculated for the entire SAR image in figure 5) is reported in table 3: the Speckle index confirms the behaviour discussed above from a visual inspection of the images. ENVI is slightly better in preserving the edges and the adaptive MCE is better in retaining the mean.

The same table reports the results obtained on an ERS image acquired over the Niger delta area in Nigeria (C-band, VV polarisation, 3-looks, the results of which are visually similar to Figure 5): again the results obtained by the ENVI gamma algorithm seem to be better than those achieved by CAESAR or the MCE method 
regarding edge preservation but not very efficient in reducing speckle. CAESAR gammann gives the Speckle index closest to the original number of looks but it does not retain the radiometric properties as well as the other two methods. For both images the segmentation results provide very low values of the ENL.

\section{Concluding discussion}

In this paper the potential of a minimum cross-entropy approach to SAR image reconstruction has been investigated. An MCE reconstruction algorithm using a novel form of the goodness-of-fit function was developed, based on the employment of an entropy function, and we used information on the local image statistics to determine the values of the regularisation parameter $\lambda$.

The validity of the MCE method is that it provides a new approach to the reconstruction problem where no assumptions are required regarding the image formation process, the nature of the image (i.e. when the cartoon model is no longer appropriate) and the expected noise probability distribution function. This can be viewed as a weakness when the image statistic can be accurately determined as happens when imaging scenes that are not too textured. However this is not always the case. Making assumptions on the probability density function for SAR images of mixed texture, such as for example dense forests and areas of relief, is not an easy task (Nezry and Yakam-Simen 1999). In this kind of situations, an approach that keeps the a priori assumptions at a minimum such as that employed in this paper is more suited (see also Dactu and Walessa 1997, Dactu et al. 1998).

The method developed was tested on simulated and real SAR data: it compared well to standard reconstruction techniques commercially available and in some cases it improved our understanding of the scene investigated. Improved results were obtained for images processed with few number of looks, i.e. when the speckle-noise is high and the knowledge of its statistics is less known. In particular good performances were achieved for images of natural landscapes, where the MCE method was found to be efficient in preserving visual texture features, such as those of forested areas.

Segmentation proved to be more effective than RCS reconstruction for images with well defined discrete areas and edges such as those found in man-made landscapes or in natural lanscapes with sharp boundaries. However it appears nonoptimal for images of natural sceneries that contain a continuous distribution of features and cover types and a mixture of textures.

In this paper the algorithm has been deliberately kept simple. The employment of edge directed windows as in (Lee 1981, Lee et al. 1999) to perform the 
local cross-entropy minimisation is likely to greatly improve the edge reconstruction, but this would introduce further assumptions on the target scene that can be problematic in certain situations, such as point targets, or areas of natural landscape with no clear boundaries. Further work should to be done in this direction to validate the MCE algorithm using very high resolution data in the presence of point targets.

Further implementations of the algorithm are also necessary to apply MCE as a routine reconstruction method. The computational time is still high compared with other commercially available filter (for a 512x512 pixels image it is about 10 times longer to run than the simulated annealing routine in CAESAR): this can be reduced, for example, by applying the adaptive MCE reconstruction in edge regions and the mean filter in uniform regions (i.e. choosing a cut-off value for $\lambda$ above which the reconstruction is not performed).

\section{Acknowledgments}

This research has been supported by an EPSRC research grant. We wish to thank Dr. Shane Cloude for interesting and helpful discussions on the topic. 


\section{References}

BURC S., GULL S. and SKILLING J., 1983, Image restoration by a powerful Maximum entropy method. Comp. Vision, Graphics and Image Proc., 23, 113-128.

DACTU M., SEIDEL K. and WALESSA M., 1998, Spatial information retrieval from remote sensing images-Part I: information theoretical perspective. IEEE Trans. Geosci. Rem. Sens., 36, N 5, 1431-1445.

DACTU M. and WALESSA M., 1997, Maximum entropy methods for despeckling and resampling synthetic aperture radar images of rough terrain. Proc. of EOS/SPIE, 3217.

GRIPPA M. and WOODHOUSE I. H., 2002, Application of Minimum CrossEntropy Methods to synthetic aperture radar images of natural landscapes. Proc PIERS02: Progress in Electromagnetic Research Symposium, Cambridge, MA, U.S.A., 1-5 July 2002.

GRIPPA M. and WOODHOUSE I. H., 2002, SAR image reconstruction using minimum cross-entropy methods. EUSARO2 (European Conference on Synthetic Aperture Radar), Köln, Germany, 4-6 June 2002.

GULL S., 1989, Developments in Maximum entropy data analysis. In Maximum entropy and Bayesian Methods (Dordrecht: Kluwer Academicc Pub.), 5371.

JAYNES E. T., 1982, On the rationale of maximum-entropy methods. Proc. IEEE, 70, N 9, 939-952.

LEE J. S. and JURKEVICH I., 1994, Speckle filtering of synthetic aperture radar images: a review. Remote Sensing Review, 8, 313-340.

LEE J. S., 1981, Refined filtering of image noise using local statistics. Comput. Vis., Graph., Image Process., 15, 380-389.

LEE J. S., GRUNES M. R. and DE GRANDI G., 1999, Polarimetric SAR Speckle Filtering and its implication for classification. IEEE Trans. Geosci. Remote Sens., 37 N 5, 2363-2373.

LOPES A. and SERY F., 1997, Optimal speckle reduction for the product model in multilook polarimetric SAR imagery and the Wishart distribution. IEEE Trans. Geosci. Remote Sens., 35, N 3, 632-647. 
McCONNEL I. and OLIVER C., 1996, Comparison of annealing and iterated filters for speckle reduction in SAR. in Microwave Sensing and Synthetic Aperture Radar (edited by G. Franceschetti et al., Proc. SPIE 2958), 7485.

NEZRY E. and YAKAM-SIMEN F., 1999, A Family of Distribution-Entropy MAP Speckle Filters for Polarimetric SAR Data and for single or MultiChannel Detected and Complex SAR Images. Proc. CEOS'99 SAR Workshop. CEOS 1999.

OLIVER C. and QUEGAN S., 1998, Understanding synthetic aperture radar images. (Arthec House Publishers).

SHI Z. and FUNG K. B., 1994, A comparison of digital speckle Filters. Proc.

IGARSS94, 8-12 August, 1994 2129-2133.

WALESSA M. and DATCU M., 2000, Model-based despeckling and information extraction from SAR images. IEEE Trans. Geosci. Rem. Sens., 38, N 5, 2258-2269.

WOODHOUSE I. H., 2001, The ratio of the arithmetic to the geometric mean: a cross-entropy interpretation. IEEE Trans. Geosci. Rem. Sens., 39, N 1, 188-189. 


\section{List of tables}

Table 1: Results for test image A and B (with $\lambda=15$ for MCE-MCE and $\lambda=100$ for $\left.\operatorname{MCE}-\chi^{2}\right)$.

Table 2: Adaptive MCE results in comparison with those obtained using CAESAR and ENVI. Refer to table 1 for the correspondent values of the original images.

Table 3: Reconstruction results for E-SAR Glen Affric data and ERS data (evaluated on the full images of $512 \times 512$ pixels).

\section{List of figures}

Figure 1: Values of $\lambda$ calculates as in equation 5 for test image A (in figure 2a) with $L=6$.

Figure 2: Test images A (a) and B (d) with added simulated 6-looks noise (b), (e) and minimum cross-entropy reconstruction (c) and (f).

Figure 3: Reconstruction of the noisy test images A and B in Fig 1 (a) and (d) using the adaptive MCE method ((a) and (e)) in comparison with ENVI Gamma ((b) and (f)), CAESAR Gammann ((c) and (g)) and CAESAR Segann ((d) and (h)) results.

Figure 4: RMSE values for test images $\mathrm{A}$ and $\mathrm{B}$ as a function of the number of looks obtained with the adaptive MCE method, ENVI and CAESAR.

Figure 5: Top: E-SAR intensity data (4-looks) over Glen Affric (a) and adaptive MCE reconstruction (b). Bottom: photograph of the same region.

Figure 6: Detail (a) of the E-SAR image in the square box in Fig. 5(a) and MCE reconstruction (b) in comparison with ENVI Gamma (c), CAESAR Gammann (d) and CAESAR Segann (e) results. 
Table 1:

\begin{tabular}{ccccc} 
& Mean & Speckle (ENL) & Edge & MSE \\
\hline TEST A, L=6 & & & & \\
\hline Original & 103.2 & 5.80 & 84.55 & - \\
Speckled & 103.4 & - & 76.38 & 2372.7 \\
\hline MCE-MCE & 103.6 & 6.21 & 44.46 & 292.1 \\
MCE- $\chi^{2}$ & 103.9 & 6.12 & 42.93 & 307.9 \\
Mean & 103.2 & 5.09 & 38.58 & 342.5 \\
\hline TEST B, L=6 & & & & \\
\hline Original & 75.5 & 5.80 & 74.10 & - \\
Speckled & 75.5 & - & 69.56 & 1129.5 \\
\hline MCE-MCE & 75.6 & 5.75 & 55.91 & 271.9 \\
MCE- $\chi^{2}$ & 75.8 & 5.38 & 55.83 & 287.5 \\
Mean & 75.5 & 4.57 & 45.05 & 300.3
\end{tabular}


Table 2:

\begin{tabular}{ccccc} 
& Mean & Speckle (ENL) & Edge & MSE \\
\hline TEST A, L=6 & \multicolumn{5}{c}{} \\
\hline Adapt. MCE & 103.7 & 7.14 & 51.75 & 235.4 \\
ENVI Gamma & 104.3 & 8.89 & 70.80 & 462.6 \\
CAESAR Gammann & 92.7 & 5.74 & 69.80 & 283.9 \\
CAESAR Segann & 103.4 & 6.65 & 84.8 & 148.83 \\
\hline TEST B, L=6 & \multicolumn{5}{c}{} \\
\hline Adapt. MCE & 76.0 & 6.86 & 56.63 & 259.6 \\
ENVI Gamma & 76.4 & 10.02 & 61.62 & 335.9 \\
CAESAR Gammann & 66.6 & 4.09 & 49.15 & 321.2 \\
CAESAR Segann & 75.41 & 4.63 & 69.8 & 283.9
\end{tabular}

Table 3:

\begin{tabular}{cccc} 
& Mean & Speckle (ENL) & Edge \\
\hline E-SAR data, L=4 & \multicolumn{3}{c}{} \\
\hline Original & 0.158 & - & 0.011 \\
Adapt. MCE & 0.159 & 8.9 & 0.015 \\
ENVI Gamma & 0.147 & 11.2 & 0.013 \\
CAESAR Gammann & 0.130 & 4.4 & 0.014 \\
CAESAR Segann & 0.163 & 0.3 & 0.007 \\
\hline ERS data, L=3 & \multicolumn{3}{c}{} \\
\hline Original & 0.210 & - & 0.026 \\
Adapt. MCE & 0.210 & 7.3 & 0.014 \\
ENVI Gamma & 0.207 & 10.1 & 0.018 \\
CAESAR Gammann & 0.173 & 3.9 & 0.014 \\
CAESAR Segann & 0.229 & 0.2 & 0.010
\end{tabular}




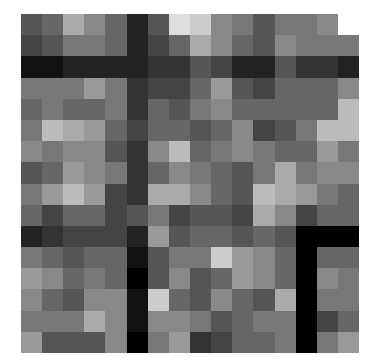

Figure 1:

(a) TEST A

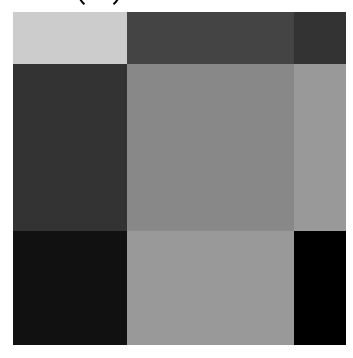

(d) TEST B

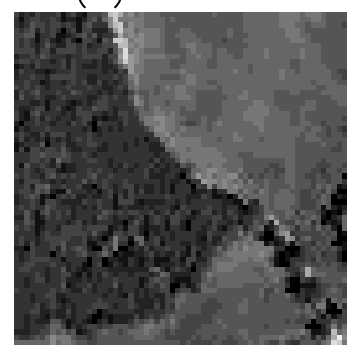

(b) Speckled

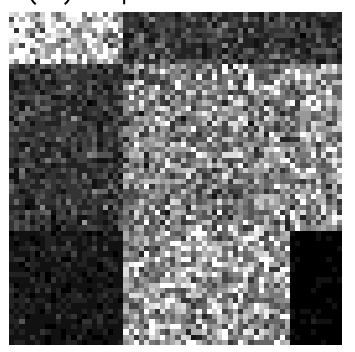

(e) Speckled

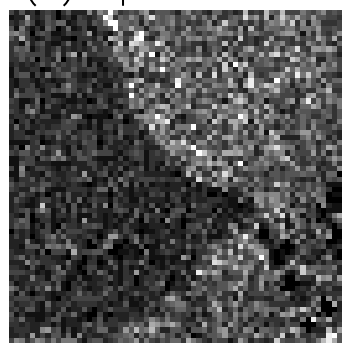

Figure 2: (c) MCE-MCE

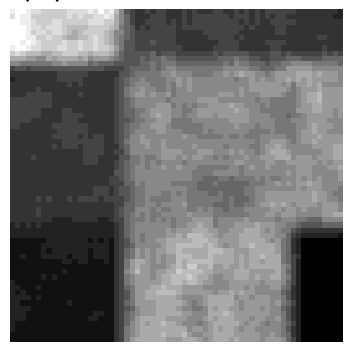

(f) MCE-MCE

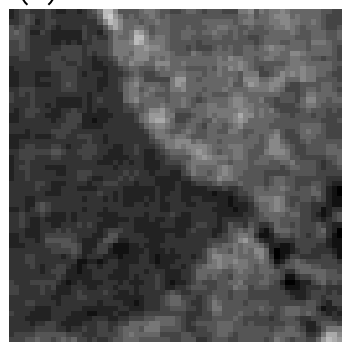



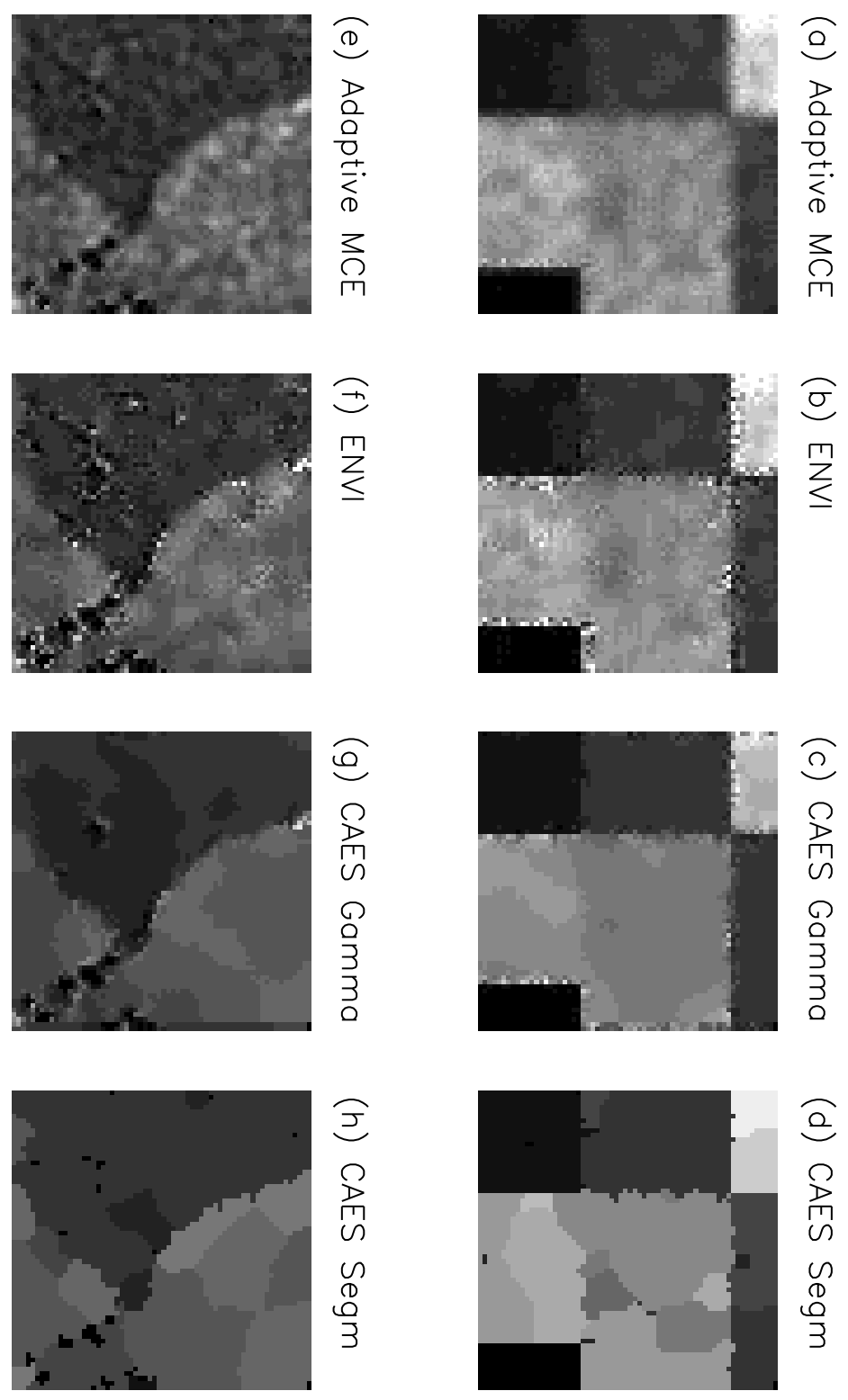

Figure 3: 

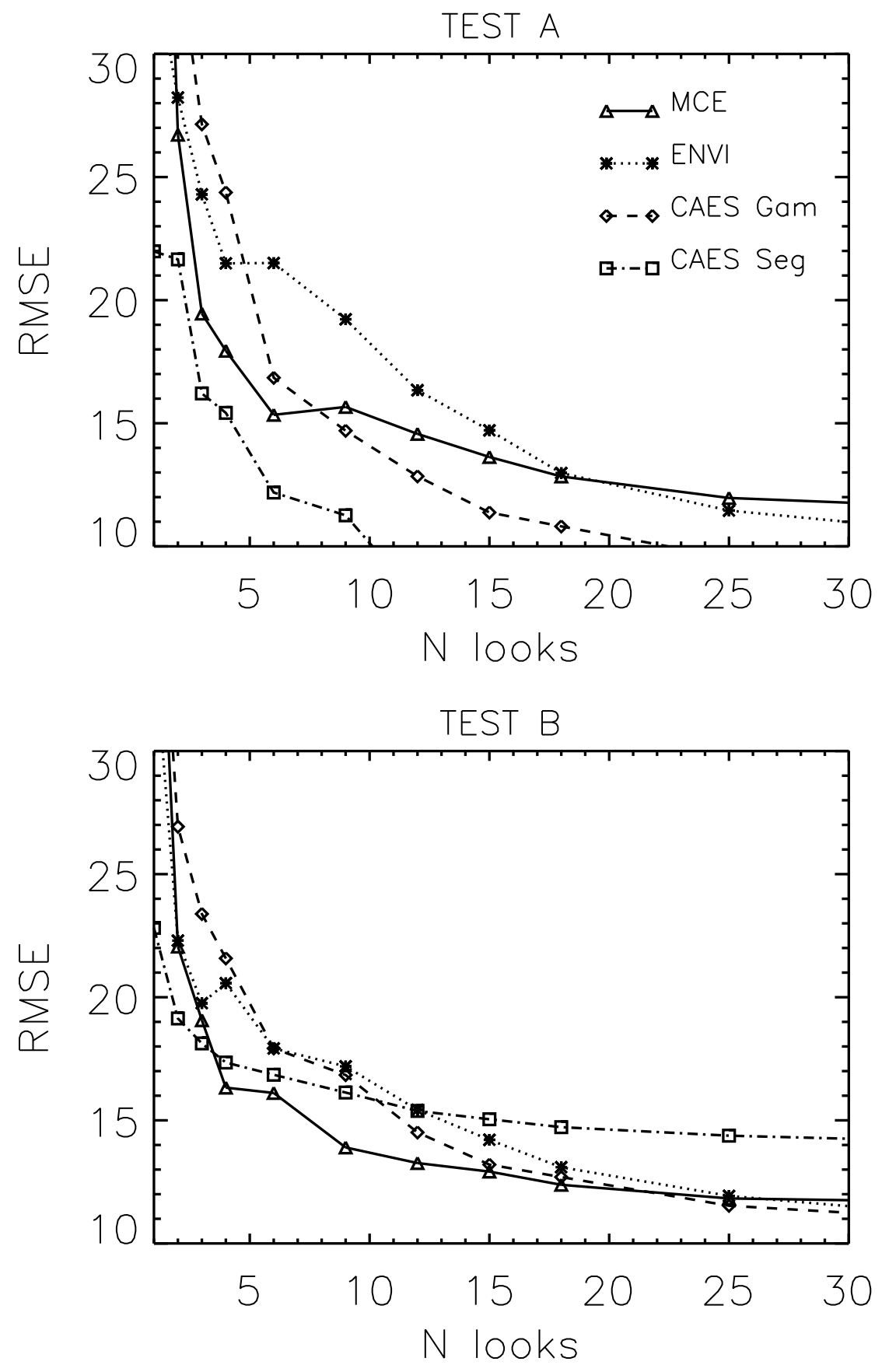

Figure 4: 
(a) ORIGINAL

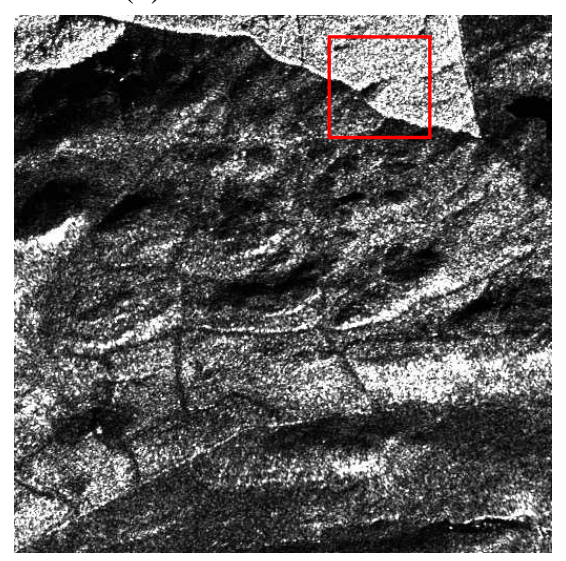

(b) Adapt. MCE

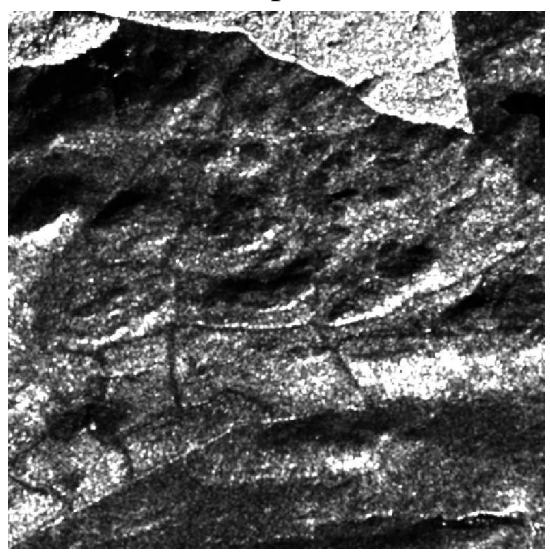

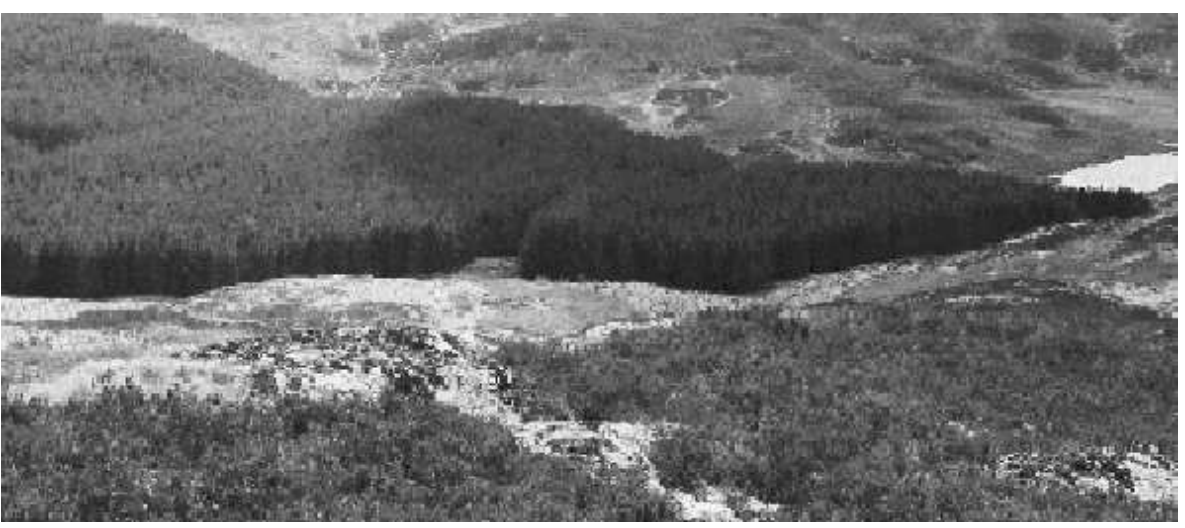

Figure 5: 
(a) ORIGINAL

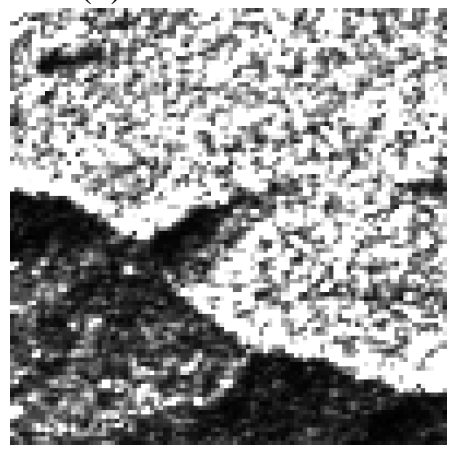

(c) ENVI Gamma

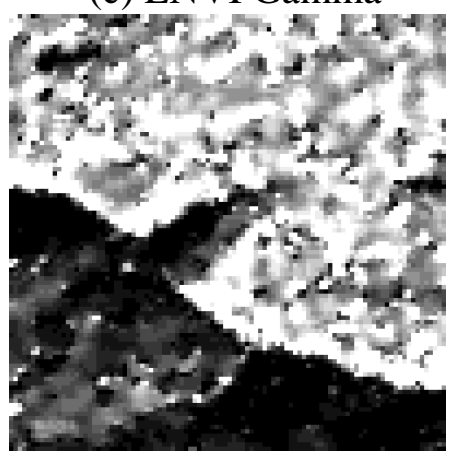

(b) Adapt. MCE

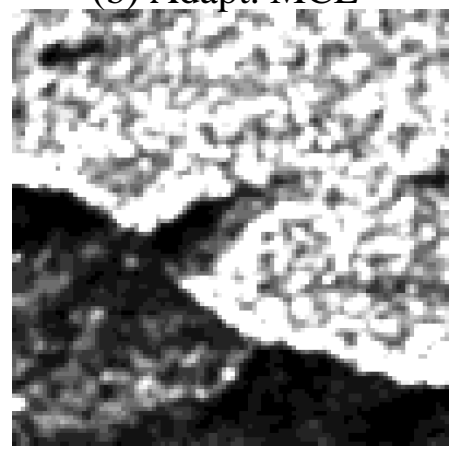

(d) CAESAR Gamma

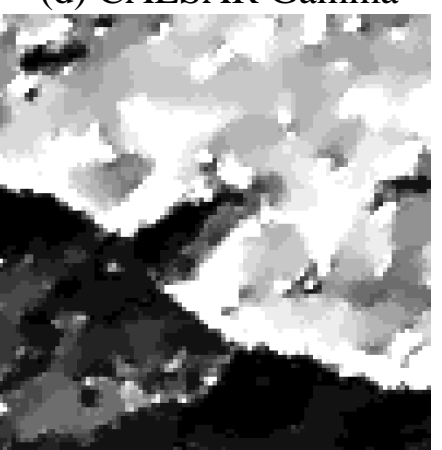

(e) CAESAR Segment

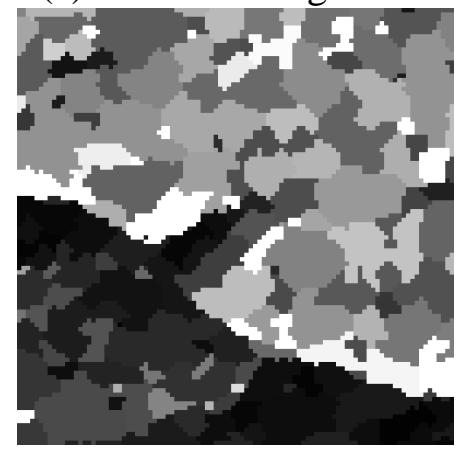

Figure 6: 